\title{
The battle against influenza: The role of neuraminidase inhibitors in children
}

\author{
Upton Allen MBBS MSc FAAP FRCPC
}

$\mathrm{I}^{\mathrm{n}}$ nfluenza viruses continue to be a major cause of morbidity and mortality worldwide. During outbreaks of influenza, the highest attack rates are among school-aged children. Secondary spread occurs to adults and other children in the family. The secondary spread is facilitated by viral shedding, which can be quite prolonged in young children (1). In neonates, influenza has been associated with significant morbidity, including a sepsis-like syndrome, apnea and lower respiratory tract disease. Children younger than five years of age have the second highest rates of infection with influenza and hospitalization, which are exceeded only by persons older than 65 years of age $(1,2)$. Increased rates of hospitalization during influenza seasons have been observed among previously healthy infants and young children (3). Besides admissions to hospitals, influenza accounts for a substantial number of outpatient visits and antibiotic prescriptions in children $(4,5)$.

Given the burden of influenza infections in children despite the medical community's best efforts at prevention, treatment strategies are required for certain groups of children, including children at risk of severe complications from influenza. Among the traditional therapeutic agents, only amantadine (Symmetrel, DuPont Pharma, Canada) is approved for treatment in children, and neither amantadine nor rimantadine (Flumadine, Forest Laboratories Inc, USA; not approved for use in Canada) is effective against influenza $B$. The newly approved neuraminidase inhibitors are active against both influenza A and B (6-8). While the bulk of the evidence for the effectiveness of the neuraminidase inhibitors has been derived from studies in adults and children older than 12 years of age, on-going studies are addressing the role of these agents in the management of influenza infections in young children.

\section{INFLUENZA VIRUS NEURAMINIDASE}

Neuraminidase is a surface glycoprotein that has enzymatic activity essential for the replication of influenza $A$ and B (8). The enzyme catalyses the cleavage of the $\alpha$-ketosidic linkage that exists between a terminal sialic acid, $N$-acetyl neuraminic acid and an adjacent sugar residue. This action has a number of important effects that enable the spread of the virus within the respiratory tract. These effects include the release of the virus from infected cells, the prevention of viral aggregates after release from host cells, the prevention of viral inactivation and the promotion of viral penetration into respiratory cells.

\section{NEURAMINIDASE INHIBITORS}

Neuraminidase inhibitors were developed to capitalize on the essential role of neuraminic acid in influenza virus replication. The drugs are sialic acid analogues, four of which, Neu5Ac2en, zanamivir (Relenza, Glaxo Wellcome Inc), oseltamivir (Tamiflu, Hoffmann-La Roche) and RWJ-270201, have been used in clinical trials (6). Neu5Ac2en was the first of the sialic acid analogues to be developed (9). The newer generation of drugs allows for a more selective and potent inhibition of influenza A and B virus neuraminic acid. Currently, two agents (zanamivir and oseltamivir) are approved for the treatment of influenza virus infections $(10,11)$. Zanamivir and oseltamivir differ from amantadine and rimantadine in terms of the types of influenza virus that they inhibit, the route of administration and the approved indications (Table 1) (12). Because the drugs target a different site than amantadine and rimantadine, zanamivir and oseltamivir inhibit the replication of influenza viral strains that are resistant to amantadine and rimantadine.

Zanamivir: Zanamivir was the first of the new generation of neuraminidase inhibitors to reach clinical trials $(7,13)$. The oral bioavailability of zanamivir is very poor (less than $5 \%)(13,14)$. Following intravenous dosing, the plasma $\mathrm{T}_{1 / 2}$ elim of zanamivir is in the region of $1.6 \mathrm{~h}$, with about $90 \%$ eliminated unchanged via urine. After inhalation of the dry powder, $15 \%$ of the drug is deposited in the lower respiratory tract, and the rest remains in the oropharyngeal region. The bioavailability averages about $20 \%$, with low blood levels. 
TABLE 1

A comparison of antiviral agents used for influenza

\begin{tabular}{|c|c|}
\hline \multicolumn{2}{|c|}{ Influenza viruses inhibited } \\
\hline \multicolumn{2}{|c|}{ Amantadine and rimantadine* inhibit influenza A only, while zanamivir and oseltamivir inhibit both influenza A and B } \\
\hline \multicolumn{2}{|c|}{ Route of administration } \\
\hline Amantadine & Oral (tablet, capsule, syrup) \\
\hline Rimantadine & Oral (tablet, syrup) \\
\hline Zanamivir & $\begin{array}{l}\text { Oral inhalation; a specially designed plastic oral inhalation device (Diskhaler, Glaxo Wellcome Inc, Canada) administers } \\
\text { zanamivir. Delivery via a nebulizer has been evaluated in children }\end{array}$ \\
\hline \multicolumn{2}{|c|}{ Ages for which treatment is approved } \\
\hline Amantadine & 1 year and older \\
\hline Rimantadine & 14 years and older \\
\hline Zanamivir & 12 years and older \\
\hline Oseltamivir & 18 years and older \\
\hline
\end{tabular}

Are the agents approved for prophylaxis?

Amantadine and rimantadine are approved for children 1 year and older

Zanamivir and oseltamivir are not approved for prophylaxis

Additional information

Because the role of neuraminidase inhibitors in children is an evolving subject, new information will, most likely, be available in the near future. Physicians should refer to current product monographs for actual dosages of the neuraminidase inhibitors. The trade names of the various agents are as follows: amantadine (Symmetrel, DuPont Pharma); rimantadine (Flumadine, Forest Laboratories Inc, USA; not approved for use in Canada); zanamivir (Relenza, Glaxo Wellcome Inc); oseltamivir (Tamiflu, Hoffmann-La Roche)

*Not approved for use in Canada

Oseltamivir: The active product of oseltamivir, GS4071, has an antiviral spectrum that is similar to that of zanamivir $(7,14)$. Oral oseltamivir is cleaved to the active drug GS4071 by esterases in the gastrointestinal tract and blood. The bioavailability of GS4071 is in the region of $80 \%(7,14)$. Maximum plasma concentrations are achieved within 3 to $4 \mathrm{~h}$ after oral administration. As with zanamivir, the blood levels of the active drug are low. However, as with zanamivir, viral inhibition occurs at relatively low concentrations. Following oral administration, the plasma $\mathrm{T}_{1 / 2}$ elim of GS4071 is about 7 to 9 h. GS4071 and the prodrug are eliminated unchanged via the kidneys.

Both zanamivir and oseltamivir appear to be free of major end organ toxicity in humans. Caution is advised if zanamivir is given to patients with underlying chronic respiratory disease because of the potential for bronchospasm $(11,12)$. No significant drug interactions with zanamivir have been seen. Coadministration of oseltamivir and probenecid results in a reduction in the clearance of GS4071 by about 50\%, with a corresponding twofold increase in plasma levels of GS4071 $(10,15)$. Nausea and vomiting are the most frequently observed adverse events in clinical trials involving oral oseltamivir. Overall, gastrointestinal side effects are mild; they may diminish if oseltamivir is taken with food and with continued administration of the drug.

\section{INDICATIONS AND CLINICAL USES}

Zanamivir: In Canada, zanamivir is indicated for the treatment of uncomplicated, acute illness due to influenza virus in patients 12 years and older who have been symptomatic for no more than $48 \mathrm{~h}$. In two of three pivotal clinical studies, there was a statistically significant reduction in the median time to the improvement of influenza symptoms in patients treated with zanamivir compared with placebo.

The recommended dosage of zanamivir is two inhalations (one $5 \mathrm{mg}$ blister per inhalation for a total dose of $10 \mathrm{mg}$ ) twice daily, approximately $12 \mathrm{~h}$ apart for five days (11). If possible, two doses should be taken on the first day of treatment, provided that there is at least $2 \mathrm{~h}$ or more between doses (16). On subsequent days, doses should be given approximately $12 \mathrm{~h}$ apart. Oseltamivir: In Canada, the approved indication for the use of oseltamivir is the treatment of uncomplicated illness due to influenza infection in adults who have been symptomatic for no more than $48 \mathrm{~h}(10)$. This indication is based on two phase III clinical trials in adults, the majority of whom had influenza A infection. No data support the efficacy of the drug in patients who started treatment after $40 \mathrm{~h}$ following the onset of symptoms (10).

The oral dosage of oseltamivir is $75 \mathrm{mg}$ twice daily for five days (10). The drug may be taken with or without food. Administration with food may improve tolerability in some patients, while at the same time slightly delaying the absorption without affecting the overall bioavailability.

\section{PAEDIATRIC DATA}

Zanamivir: Published data on the efficacy and safety of zanamivir are based primarily on clinical trials involving patients 12 years of age and older (17-20). No differences in safety and efficacy were observed between children 12 to 16 years of age and young adults. Data are limited for children younger than 12 years of age. A randomized, double-blind, placebo controlled trial was conducted in 471 paediatric patients aged five to 12 years (21). All patients were instructed in the use of the Diskhaler (Glaxo Wellcome Inc, Canada), and assistance was provided when necessary. The results showed that there was a 1.25 day reduction in clinically significant symptoms of influenza. However, in the intent-totreat analysis, the reduction in symptoms was 0.5 days. The 
adverse events reported were generally mild or moderate in severity, and similar to those reported for patients receiving placebo.

Infants and young children with influenza require a different delivery system than that used for older individuals. Such a system will, most likely, involve nebulization. In an open label study, zanamivir was well tolerated when given as single inhalations of $10 \mathrm{mg}$ via a nebulizer or the Diskhaler to children three months to 12 years of age (22). The systemic exposure in these children was found to be comparable with the exposure observed in healthy adult volunteers using the same dosage and formulation (22).

Oseltamivir: Published data on the safety and efficacy of oseltamivir are limited for children. The current indication for the use of the drug is based on clinical trials in adults (between 18 and 65 years of age inclusive) (23-26). Pharmacokinetic data show that the steady state exposure of $2 \mathrm{mg} / \mathrm{kg}$ twice daily for five days across the age range of one to 12 years is similar to the range of exposures associated with safety and efficacy in adults ( $75 \mathrm{mg}$ twice daily) (27).

Oseltamivir is available as a $75 \mathrm{mg}$ capsule for oral use twice daily. Capsules may not be ideal for young children who are very ill. A liquid formulation was shown to be safe and well accepted by healthy children one to 12 years of age and children with asthma six to 12 years of age (28). In a double-blind, placebo controlled, randomized trial, 698 healthy children aged one to 12 years were evaluated (29). Among 452 children with laboratory-confirmed influenza, the drug reduced the median time to alleviation of illness by $26 \%$. Median time to return to normal activity was reduced by $40 \%$. The use of oseltamivir was associated with a $44 \%$ reduction in the development of acute otitis media and associated antibiotic use. The rate of discontinuation of oseltamivir due to adverse affects was low relative to placebo ( $1.8 \%$ versus $1.2 \%$, respectively).

\section{PREGNANCY}

The efficacy and safety of oseltamivir and zanamivir have not been established in pregnancy $(10,11)$. While amantadine and rimantadine have been shown to be associated with teratogenicity and reproductive toxicity in laboratory animals, the neuraminidase inhibitors have not been shown to have these effects. Both agents cross the placenta and are excreted in breast milk. Given the low systemic concentrations of zanamivir following inhalation, this agent may be preferred for use in pregnancy, especially during the second and third trimesters (6).

\section{SUMMARY}

The neuraminidase inhibitors are an important advance in the treatment of influenza infections. In contrast to amantadine and rimantadine, the neuraminidase inhibitors are active against both influenza $\mathrm{A}$ and $\mathrm{B}$. Oseltamivir and zanamivir are not approved for the prevention of influenza infection. Data are limited in children younger than 12 years of age. Safety profiles appear promising with respect to the potential use of these agents in children. However, both agents are more expensive than amantadine and rimantadine. This is particularly important given the extent of the clinical gain re- ported in trials. In this regard, cost effectiveness considerations will feature prominently as physicians consider the role of these agents in the treatment of influenza infections in healthy children. Firm paediatric recommendations cannot be made at this time because of the lack of data. Current recommendations, therefore, remain structured around the use of amantadine and rimantadine. Thus, at the present time, therapy for influenza virus infection should be considered for children at increased risk for severe or complicated influenza infection, otherwise healthy children with a severe illness, and persons with very special environmental, family or social situations. In these situations, zanamivir or oseltamivir may be used if the child is of the appropriate age (Table 1).

\section{REFERENCES}

1. American Academy of Pediatrics. Influenza. In: Pickering LK, ed. 2000 Red Book: Report of the Committee on Infectious Diseases, 25th edn. Elk Grove Village: American Academy of Pediatrics, 2000:351-9.

2. LCDC. Influenza in Canada - 1998-1999 season. Can Commun Dis Rep 1999;25:1-8.

3. Izurieta HS, Thompson WW, Kramarz P, et al. Influenza and the rates of hospitalization for respiratory disease among infants and young children. N Engl J Med 2000;342:232-9.

4. Neuzil KM, Mellen BG, Wright PF Jr, Mitchell EF, Griffin MR. The effect of influenza on hospitalizations, outpatient visits, and courses of antibiotics in children. $\mathrm{N}$ Engl J Med 2000;342:225-31.

5. Glezen WP. Influenza and hospitalizations in children. N Engl J Med 2000;342:1752-53.

6. Gubareva LV, Kaiser L, Hayden FG. Influenza virus neuraminidase inhibitors. Lancet 2000;355:827-35.

7. Bardsley-Elliot A, Noble S. Oseltamivir. Drugs 1999;58:851-60.

8. Calfee DP, Haydon FG. New approaches to influenza chemotherapy. Neuraminidase inhibitors. Drugs 1998;56:537-553.

9. Meindl P, Tuppy H. 2-doexy-2-3-dehydroxialic acids I. Synthesis and properties of 2-deoxy, 3 dehydro- $N$-acylneuraminic acids and their methyl esters. Monatshefte für Chemie 1969;100:1295-1306.

10. Hoffmann-La Roche Limited. Product Monograph, Oseltamivir Phosphate (Tamiflu). Nutley: Hoffman-La Roche Limited, 1999.

11. Glaxo Wellcome Inc. Product Monograph, Zanamivir (Relenza). Research Triangle: Glaxo Wellcome Inc, 1999.

12. Neuraminidase inhibitors for treatment of influenza A and B infections. MMWR Morb Mortal Wkly Rep 1999;48:1-9.

13. Waghorn SL, Goa KL. Zanamivir. Drugs 1998;55:721-5.

14. Hayden FG. Antiviral drugs (other than antiretrovirals). In: Mandell GL, Bennett JE, Dolin E, eds. Mandell, Douglas, and Bennett's Principles and Practice of Infectious Diseases, 5th edn. Philadelphia: Churchill Livingstone, 2000:481-91.

15. He G, Massarella J, Aitken M, et al. The pharmacokinetics and safety of the oral neuraminidase inhibitor Ro 64-0796/GS4104 when administered concurrently with cimetidine or probenecid in healthy subjects. J Antimicrob Chemother 1999;44(Suppl A):44. (Abst)

16. Cada DJ. Zanamivir. In: Drug Facts and Comparisons 2000, 54th edn. St Louis: Facts and Comparisons, 1999:1422-3.

17. Hayden FG, Osterhaus AD, Treanor JJ, et al. Efficacy and safety of the neuraminidase inhibitor zanamivir in the treatment of influenza virus infections. N Engl J Med 1997;337:874-80.

18. Randomised trial of efficacy and safety of inhaled zanamivir in treatment of influenza A and B virus infections. The MIST (Management of Influenza in the Southern Hemisphere Trialists) Study Group. Lancet 1998;352:1877-81.

19. Monto AS, Fleming DM, Henry D, et al. Efficacy and safety of the neuraminidase inhibitor zanamivir in the treatment of influenza A and B virus infections. J Infect Dis 1999;180:254-61.

20. Monto As, Robinson DP, Herlocher ML, Hinson JM, Elliot MJ, Crisp A. Zanamivir in the prevention of influenza among healthy adults: A randomized controlled trial. JAMA 1999;282:31-5. 
21. Hendrick JA, Barzilai A, Behre U, et al. Zanamivir for treatment of symptomatic influenza A and B infection in children five to twelve years of age: a randomized controlled trial. Pediatr Infect Dis J 2000;19:410-7.

22. Peng AW, Hussey EK, Rosolowski B, et al. Pharmacokinetics and tolerability of a single inhaled dose of zanamivir in children. Curr Ther Res Clin Exp 2000;61:33-46.

23. Treanor JJ, Hayden FG, Vrooman PS, et al. Efficacy and safety of the oral neuraminidase inhibitor oseltamivir in treating acute influenza: A randomized controlled trial. US Oral Neuraminidase Study Group. JAMA 2000;283;1016-24.

24. Aoki FY, Osterhaus AD, Rimmelzwann GF, Kinnersley N, Ward P. Oral GS4104 successfully reduces duration and severity of naturally acquired influenza. Interscience Conference on Antimicrobial Agents and Chemotherapy. San Diego, September 24 to 27,1998 . (Abst)

25. Oxford JS, Ward P, Kinnersley N. Influenza treatment with oral oseltamivir reduces viral load and antibiotic use related to respiratory complications. Eur Respir J 1999;14:254s. (Abst)

26. Nicholson KG, Ward P, Kinnersley N. Oral GS4104 in the treatment of influenza in adults is effective and reduces influenza-related complications and need for antibiotic treatment. J Antimicrob Chemother 1999;44:41-2. (Abst)

27. Oo C, Barrett J, Mann J, Dorr A, Ward P. Pharmacokinetics of the oral neuraminidase inhibitor oseltamivir in pediatric patients. Clin Microbiol Infect 2000;6(Suppl 1):202. (Abst)

28. Whitley R, Young N, Ipe D, Mils R, Dutkowski R, Ward P. Safety and acceptability of oseltamivir liquid formulation in the treatment of influenza in children aged one to 12 years. 9th International Congress on Infectious Diseases. Buenos Aires, April 10 to 13, 2000.

29. Reisinger K, Hayden F, Whitley R, et al. Oral oseltamivir is effective and safe in the treatment of children with acute influenza. Clin Microbiol Infect 2000;6(Suppl 1):202. (Abst)

\section{INFECTIOUS DISEASES AND IMMUNIZATION COMMITTEE}

Members: Drs Upton Allen, The Hospital for Sick Children, Toronto, Ontario (principal author); H Dele Davies, Division of Infectious Diseases, Alberta Children's Hospital, Calgary, Alberta; Joanne Embree, The University of Manitoba, Winnipeg, Manitoba (chair); Mireille Lemay, Department of Infectious Diseases, Sainte-Justine Hospital, Montréal, Québec; Gary Pekeles, The Montreal Children's Hospital, Montreal, Quebec (director responsible)

Consultants: Drs Noni MacDonald, Faculty of Medicine, Dalhousie University, Halifax, Nova Scotia; Victor Marchessault, Cumberland, Ontario Liaisons: Drs Susan King, Division of Infectious Diseases, The Hospital for Sick Children, Toronto, Ontario (Canadian Paediatric AIDS Research Group); Scott Halperin, Department of Pediatrics, IWK-Grace Health Centre, Halifax, Nova Scotia (IMPACT); Monique Landry, Direction de la santé publique de Laval, Laval, Québec (Public Health); Larry Pickering, Centre for Pediatric Research, Norfolk, Virginia (American Academy of Pediatrics); John Waters, Alberta Health, Edmonton, Alberta (Epidemiology)

This article also appears in Paediatr Child Health 2000;5(8):457-460. 


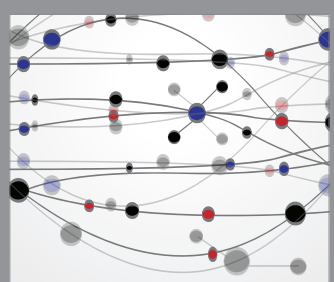

The Scientific World Journal
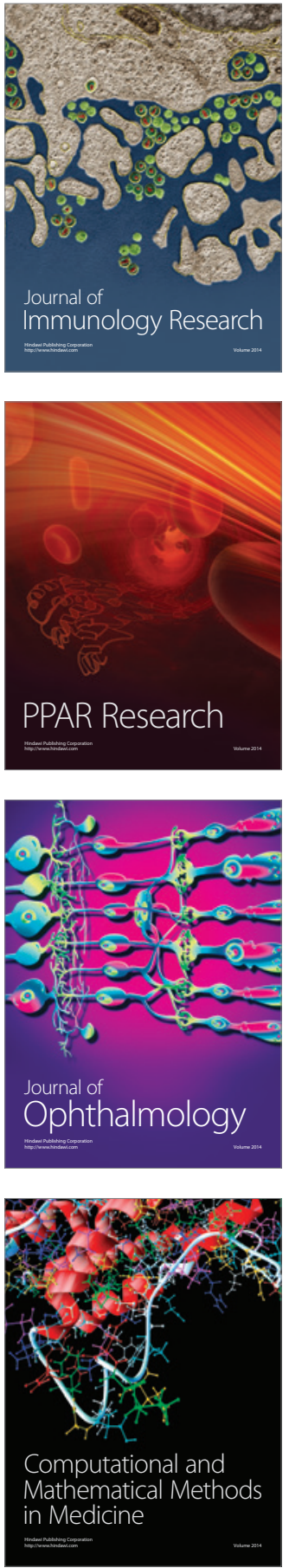

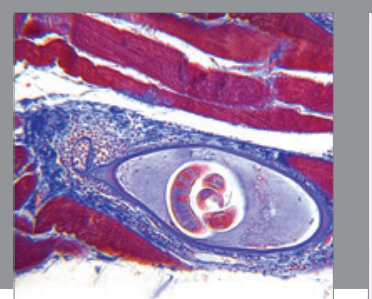

Gastroenterology Research and Practice

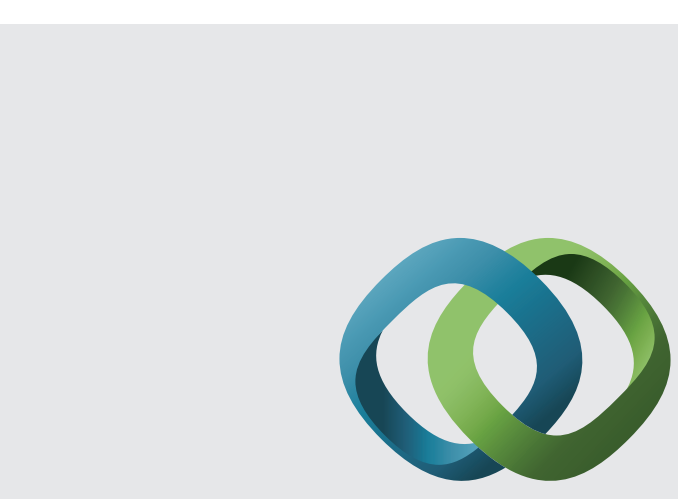

\section{Hindawi}

Submit your manuscripts at

http://www.hindawi.com
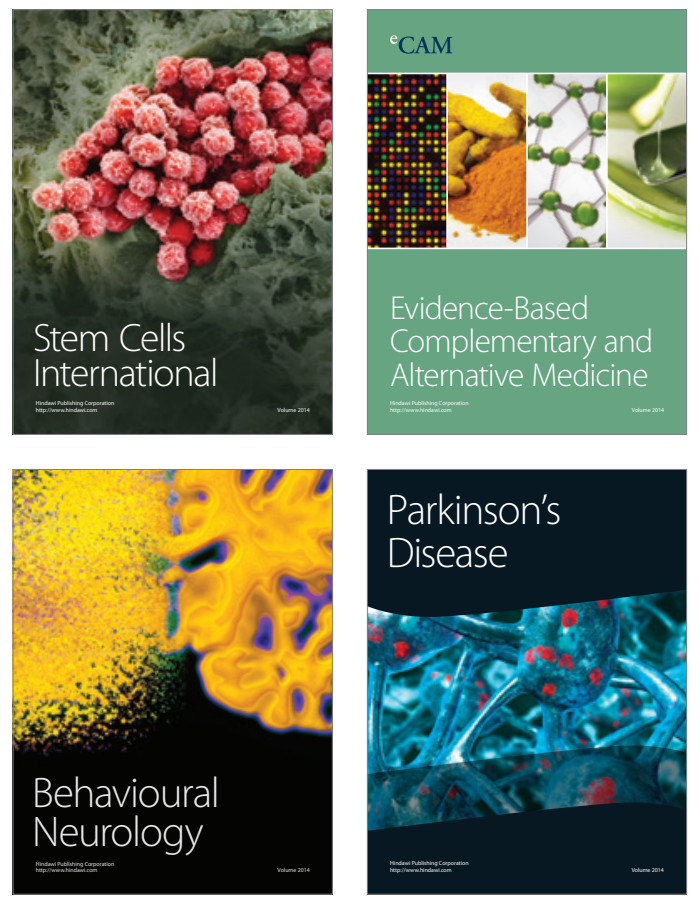
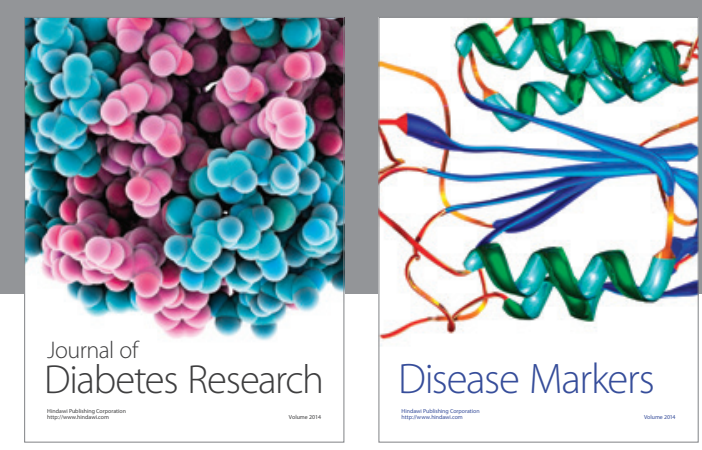

Disease Markers
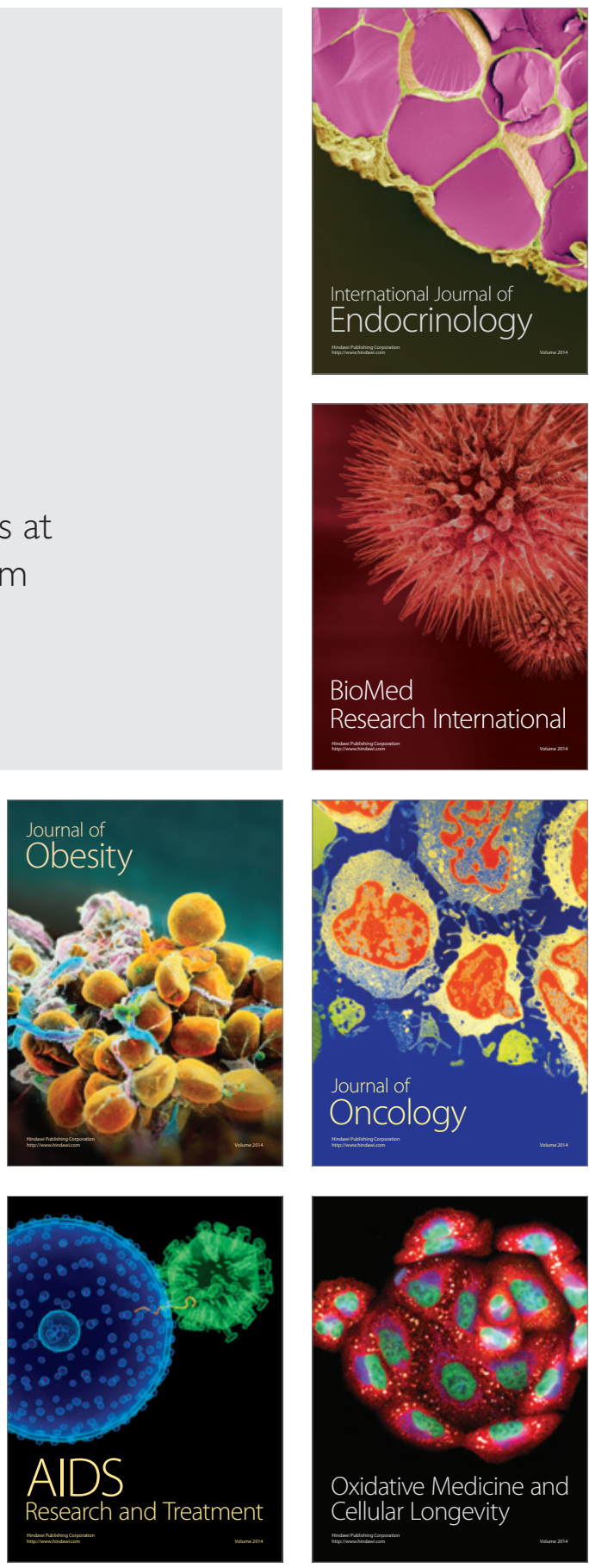